\title{
Non-selective beta blockers in cirrhosis: time to extend the indications?
}

\author{
Dev Katarey, Rajiv Jalan
}

Liver Failure Group, UCL Institute for Liver and Digestive Health, UCL Medical School, Royal Free Hospital, London, UK Correspondence to: Rajiv Jalan. Liver Failure Group, UCL Institute for Liver and Digestive Health, UCL Medical School, Royal Free Hospital, Pond Street, London NW3 2QG, UK. Email: r.jalan@ucl.ac.uk.

Provenance: This is an invited article commissioned by the Academic Editor Dr. Wei Liu (Department of Gastroenterology of Yichang Central People's Hospital. Institute of Digestive Disease, China Three Gorges University, Yichang, China).

Comment on: Villanueva C, Albillos A, Genescà J, et al. $\beta$ blockers to prevent decompensation of cirrhosis in patients with clinically significant portal hypertension (PREDESCI): a randomised, double-blind, placebo-controlled, multicentre trial. Lancet 2019;393:1597-608.

Submitted Aug 30, 2019. Accepted for publication Sep 07, 2019.

doi: $10.21037 /$ atm.2019.09.56

View this article at: http://dx.doi.org/10.21037/atm.2019.09.56

In a cirrhotic patient, irrespective of etiology, the index decompensation represents a critical landmark in their disease progression. Life expectancy plummets with 2 -year survival as low as $50 \%$ after the development of ascites, which also happens to be the most common decompensating event (1). It is imperative that liver transplantation becomes a therapeutic consideration at this point, however liver transplantation is not without its own morbidity and mortality. It is thereby of vast importance to prevent index decompensation in patients with compensated cirrhosis. A recent paper published in The Lancet, the "PREDESCI" trial, describes work done by a Spanish group which reports the results of a randomised and controlled clinical trial of non-selective beta blockers (NSBB) in preventing or delaying index decompensation (2). Decompensation was defined as the development of ascites (grade 2 or 3), bleeding related to portal hypertension, and hepatic encephalopathy.

The investigators randomised 201 participants who were recruited between January 2010 to July 2013. Prior to randomisation all participants underwent measurement of hepatic venous pressure gradient (HVPG) and only patients with an HVPG of $\geq 10 \mathrm{mmHg}$ were included. Included patients were administered IV propranolol and their dynamic response was ascertained-those with a $\geq 10 \%$ reduction in their HVPG were classed as "responders", and $\leq 10 \%$ reduction were classed as "non-responders". Responders ( $\mathrm{n}=135)$ were randomised to propranolol $(\mathrm{n}=67)$ or placebo $(\mathrm{n}=68)$, and non-responders $(\mathrm{n}=66)$ to carvedilol $(n=33)$ or placebo $(n=33)$. The primary endpoint was the incidence of decompensation or death.

The primary endpoint of decompensation or death occurred in $16(16 \%)$ participants in the NSBB group versus 27 (27\%) in the placebo group (HR 0.51; 95\% CI, $0.26-0.97, \mathrm{P}=0.041)$. This difference was predominantly due to significant reduction in new-onset ascites seen in the NSBB group (20\% versus 9\%, HR 0.42; 95\% CI, 0.19-0.92, $\mathrm{P}=0.030)$. The rates of portal hypertension-related bleeding ( $3 \%$ versus $4 \%$, HR $1.52 ; 95 \% \mathrm{CI}, 0.34-6.82, \mathrm{P}=0.61$ ), hepatic encephalopathy ( $5 \%$ versus $4 \%$, HR $0.92 ; 95 \%$ CI, $0.40-2.21, \mathrm{P}=0.98)$, and death (11\% versus $8 \%$, HR 0.54 ; $95 \% \mathrm{CI}, 0.20-1.48, \mathrm{P}=0.23$ ) were similar in both groups.

\section{Does the generated data change clinical practice?}

This was a well-designed and well-executed, randomised, double-blind, placebo-controlled, multicentre trial performed by leaders in the field, which provides an evidence-base for a question of paramount importance. However, despite being a well-executed trial with an attractive result, there are numerous considerations which need to be made before applying these results to our everyday practice.

It is important to note that the intervention and placebo groups only diverge in decompensation-free survival after 2 years of follow-up. This occurred in groups that achieved extraordinary compliance to therapy with 83 participants $(82 \%)$ in the NSBB group maintaining adequate adherence 


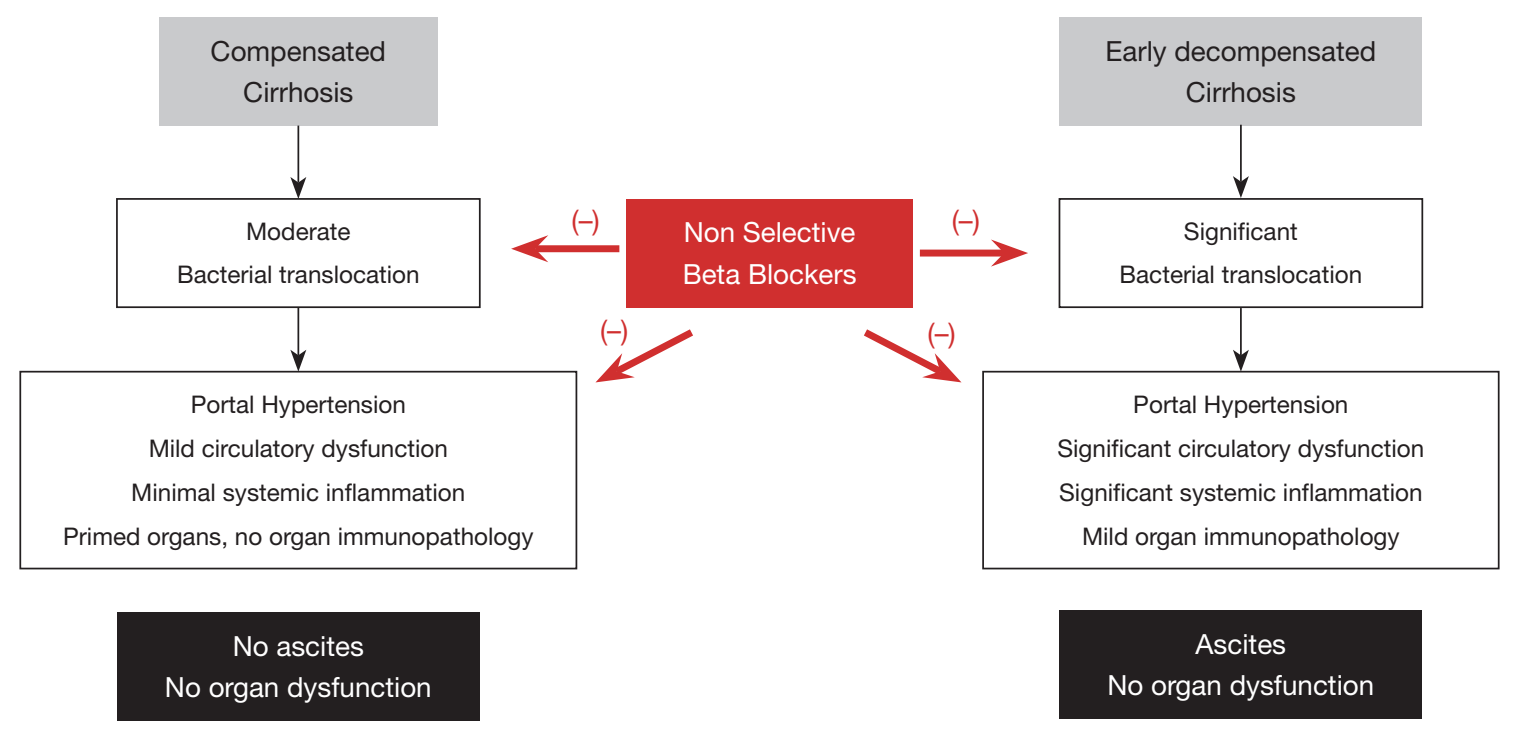

Figure 1 The effect of non-selective beta blockers on reducing bacterial translocation and thereby systemic inflammation, portal hypertension, and decompensation.

as evidenced by pill counting. This degree of compliance is extremely rare in clinical practice and therefore puts into question the applicability of these results in a real-world situation.

Furthermore, this divergence in the risk of developing ascites, which surprisingly was the main event that differed between the two groups, remains an unexplained phenomenon. The authors state that HVPG progressively declined at each visit compared with baseline, but this is not seen in the data presented. A significant reduction in HVPG was noted at 12 months but this plateaued afterwards thereby bringing into question whether it was the reduction HVPG that was mechanistically related to a reduction in the risk of developing ascites or whether these are 'mere' associations. Importantly, no effect on decompensation or death was observed for NSBB therapy in a subgroup analysis of those with a HVPG $\geq 16 \mathrm{mmHg}$, bringing into question the idea that HVPG reduction was the main driver of the improved decompensation-free survival identified. Alternate hypotheses for the effect of NSBB should be considered such as modulation of systemic inflammation (Figure 1). It is well-known that NSBB can affect gut motility and rates of bacterial translocation thereby impacting on systemic inflammation (3), which in turn may modulate severity of portal hypertension (4) and the development of ascites according to the revised hypothesis of hepatic decompensation in cirrhosis (5). Therefore, surrogate and sensitive markers of inflammation, bacterial translocation and circulatory dysfunction may have provided more pathophysiological insights into the underpinning mechanism behind improved decompensation-free survival after two years.

It is also important to note that the aetiology of liver disease for participants in this study is not reflective of many national populations. In this cohort, hepatitis $\mathrm{C}$ virus (HCV) accounted for $56 \%$ of total participants whereas alcohol and non-alcoholic steatohepatitis (NASH) only accounted for $16 \%$ and $6 \%$ of the cohort, respectively. In most Western populations, alcohol and NASH would be exceedingly common etiologies for cirrhosis $(6,7)$. In PREDESCI, no significant effect of NSBBs were observed on decompensating events in patients with alcoholrelated cirrhosis. Even in populations where $\mathrm{HCV}$ is more prevalent, it is important to take into account that this study pre-dates the era of direct-acting antiviral (DAA) agents, which can reduce HVPG and also prevent decompensation (8). Given the superior efficacy of the DAAs versus interferon-based regimens we can expect that their use would reduce the efficacy of NSBBs in preventing decompensation if the PREDESCI trial were it to be repeated today.

The recruitment methodology used in this study required measurement of HVPG for patient selection, which is only available in a small number of centres. Without any reliable 
non-invasive measures of HVPG, the notion of blanket HVPG measurements in a compensated cirrhosis cohort would translate to enormous healthcare resource utilisation which, of course, has substantial financial and logistical implications. An alternative may be to treat all compensated cirrhosis patients with carvedilol. Unfortunately, many patients would be treated below the HVPG threshold of $10 \mathrm{mmHg}$ and some for HVPG $\geq 16 \mathrm{mmHg}$ for which there is simply no evidence. The authors report that the reduction in decompensation when given NSBBs were mostly seen in those with small varices (14\% versus $34 \%$, HR 0.45 ; $95 \% \mathrm{CI}, 0.20-0.98)$ but it is unclear whether the presence of small varices can act as a surrogate marker of HVPG to then guide prescribing or withholding NSBB therapy.

It is also interesting to note that this population demonstrated a low incidence of decompensation compared with previous studies, and the reasons for this are unclear. Ripoll et al. describe individuals with an untreated HVPG $\geq 10 \mathrm{mmHg}$ having nearly a $20 \%$ incidence of decompensation versus $\leq 10 \%$ in the PREDESCI trial at 18 months (9). This is important to consider because the magnitude of effect of NSBBs in the PREDESCI trial would be diluted by this lower rate of decompensation in the placebo group.

\section{Conclusions}

Villanueva et al. should be commended for their excellent study (2). It lays the evidence-based foundation for a topic which should concern all hepatologists-preventing index decompensation. We feel that the PREDESCI study provides credence to prescribing NSBB therapy to untreated compensated $\mathrm{HCV}$ cirrhosis patients with small varices at screening endoscopy. Whether the DAAtreated HCV compensated cirrhosis patients will benefit is unknown. Other aetiologies may be prospectively confirmed for this indication after the results of a further study, which is currently ongoing in the UK, namely, "Beta-blockers or placebo for primary prophylaxis of oesophageal varices (BOPPP)" (10) are available.

\section{Acknowledgments}

None.

\section{Footnote}

Conflicts of Interest: R Jalan has served as a speaker, a consultant and an advisory board member for Sequana Medical, Yaqrit, Mallinckrodt, Organovo, Prometic, Takeda, has received research funding from Yaqrit, Takeda, owns stocks and shares in Yaqrit, Thoeris, Cyberlive and owns the patent Yaq-001; DIALIVE; Ornithine Phenylacetate; TLR4 antagonist. D Katarey has no conflicts of interest to declare.

Ethical Statement: The authors are accountable for all aspects of the work in ensuring that questions related to the accuracy or integrity of any part of the work are appropriately investigated and resolved.

\section{References}

1. Ginés P, Quintero E, Arroyo V, et al. Compensated cirrhosis: natural history and prognostic factors. Hepatology 1987;7:122-8.

2. Villanueva C, Albillos A, Genescà J, et al. $\beta$ blockers to prevent decompensation of cirrhosis in patients with clinically significant portal hypertension (PREDESCI): a randomised, double-blind, placebo-controlled, multicentre trial. Lancet 2019;393:1597-608.

3. Mookerjee RP, Pavesi M, Thomsen KL, et al. Treatment with non-selective beta blockers is associated with reduced severity of systemic inflammation and improved survival of patients with acute-on-chronic liver failure. J Hepatol 2016;64:574-82.

4. Mehta G, Gustot T, Mookerjee RP, et al. Inflammation and portal hypertension - the undiscovered country. J Hepatol 2014;61:155-63.

5. Bernardi M, Moreau R, Angeli P, et al. Mechanisms of decompensation and organ failure in cirrhosis: From peripheral arterial vasodilation to systemic inflammation hypothesis. J Hepatol 2015;63:1272-84.

6. Pimpin L, Cortez-Pinto H, Negro F, et al. Burden of liver disease in Europe: Epidemiology and analysis of risk factors to identify prevention policies. J Hepatol 2018;69:718-35.

7. Younossi ZM, Stepanova M, Afendy M, et al. Changes in the prevalence of the most common causes of chronic liver diseases in the United States from 1988 to 2008. Clin Gastroenterol Hepatol 2011;9:524-30.e1; quiz e60.

8. Park H, Wang W, Henry L, Nelson DR. Impact of AllOral Direct-Acting Antivirals on Clinical and Economic Outcomes in Patients With Chronic Hepatitis C in the United States. Hepatology 2019;69:1032-45.

9. Ripoll C, Groszmann R, Garcia-Tsao G, et al. Hepatic 
Page 4 of 4

venous pressure gradient predicts clinical decompensation in patients with compensated cirrhosis. Gastroenterology 2007;133:481-8.

10. NIH National Library of Medicine - Clinical Trials.

Cite this article as: Katarey D, Jalan R. Non-selective beta blockers in cirrhosis: time to extend the indications? Ann Transl Med 2019;7(Suppl 8):S355. doi: 10.21037/atm.2019.09.56
Beta-blockers for oesophageal varices (BOPPP). Available online: https://clinicaltrials.gov/ct2/show/ NCT03776955 